\title{
A Comparative Study of the Anti-Platelet Effects of cis- and trans-Resveratrol
}

\author{
Hwa Kim ${ }^{1}$, Seok-Jeong Oh ${ }^{1}$, Yingqiu Liu ${ }^{1}$ and Moo-Yeol Lee ${ }^{1, *}$ \\ ${ }^{1}$ College of Pharmacy, Chonnam National University, Gwangju 500-757, Republic of Korea
}

\begin{abstract}
Although various biological activities of resveratrol have been extensively studied, most reports have focused on trans-resveratrol and little attention has been paid to the cis-isomer. In this study, the effect of cis-resveratrol on platelet activity was examined and compared with that of the trans-isomer. Treatment with cis-resveratrol resulted in inhibition of platelet aggregation induced by thrombin, collagen or ADP, which are representative aggregation-inducing agents, and the trans-isomer elicited the same effects. These effects were concentration-dependent in the range of 1-100 $\mu \mathrm{M}$. However, the potency of the cis-isomer was much lower than that of the trans-isomer; the $\mathrm{IC}_{50}$ values for the cis-isomer versus the trans-isomer were $31 \pm 12$ vs $15 \pm 3,16 \pm 3$ vs $9 \pm 4$, and $60 \pm 15$ vs $25 \pm 6 \mu \mathrm{M}$ for thrombin-, collagen- and ADP-induced aggregation, respectively. These results indicate that cisresveratrol has a less potent anti-platelet activity, compared with the trans-isomer, and raise the possibility that the biological activities of the cis-isomer may be different from those of the trans-isomer. It will be necessary to evaluate the activity of cis-resveratrol independently of the trans-isomer.
\end{abstract}

Key Words: Resveratrol, Platelets, Anti-platelet effect

\section{INTRODUCTION}

Resveratrol is a natural polyphenolic compound that is widely present in red wines and other dietary components such as peanuts, grapes and mulberries. It has been demonstrated to have various biological activities including free radical scavenging, anti-inflammation, vasorelaxation, induction of endothelial nitric oxide synthase, and anti-cancer activity (Zbikowska and Olas, 2000). The anti-platelet effect of resveratrol has also been extensively studied, which serves as one of the major activities related with cardiovascular protective effect (Olas and Wachowicz, 2005). Indeed, resveratrol is effective against platelet adhesion to collagen or fibrinogen and granular secretion, as well as the whole procedures of aggregation (Zbikowska et al., 1999; Olas et al., 2001b). Diverse biochemical functions have been suggested as mechanisms underlying the anti-aggregatory effect of resveratrol including the inhibition of eicosanoid synthesis and phosphoinositide hydrolysis (Zbikowska et al., 1999; Olas et al., 2005; Crescente et al., 2009), the suppression of reactive oxygen species (ROS) production (Olas et al., 1999; Olas et al., 2001a), the blocking of calcium signaling (Dobrydneva et al., 1999), the stimulation of nitric oxide generation (Gresele et al., 2008), and the modulation of signaling molecules such as protein kinase C (Yang et al., 2008b), phospholipase C (Yang et al., 2008a), p38 MAPK (Shen et al., 2007).

Resveratrol exists as either the trans-isomer [(E)-resveratrol, 5-\{(E)-2-(4-hydroxyphenyl)ethenyl\}benzene-1,3-diol; CAS Number 501-36-0] or cis-isomer [(Z)-resveratrol, 5-\{(Z)-2-(4-hydroxyphenyl)ethenyl\}benzene-1,3-diol; CAS Number 61434-67-1] (Orallo, 2006) (Fig. 1). The term resveratrol has, however, generally referred to only the trans-isomer and little attention has been paid to cis-resveratrol. As a necessary consequence, most studies were done with trans-resveratrol or with an undefined mixture of both isomers, and little is known regarding the biological activities of cis-resveratrol (Orallo, 2006). This has been the case with studies described above reporting the anti-platelet effects of resveratrol. This may have been due to the absence, until recently, of commercially available cisresverarol (Leiro et al., 2004; Orallo, 2006). Now, however, a few chemical companies have started to produce cis-isomer.

There are only a limited number of reports that have examined the biological effects of cis- versus trans-resveratrol. For instance, cis-resveratrol exhibited slightly weaker effects in www.biomolther.org

Open Access DOI: 10.4062/biomolther.2011.19.2.201

pISSN: 1976-9148 elSSN: 2005-4483

Copyright $\odot 2011$ The Korean Society of Applied Pharmacology
Received Jan 22, 2011 Revised Jan 30, 2011 Accepted Feb 7, 2011

*Corresponding Author

E-mail: mlee@dongguk.edu

Tel: +82-31-961-5222, Fax: +82-31-961-5206 
<smiles>Oc1ccc(/C=C\c2cc(O)cc(O)c2)cc1</smiles>

cis-Resveratrol<smiles>Oc1ccc(/C=C/c2cc(O)cc(O)c2)cc1</smiles>

Fig. 1. Chemical structures of cisand trans-resveratrol.

A
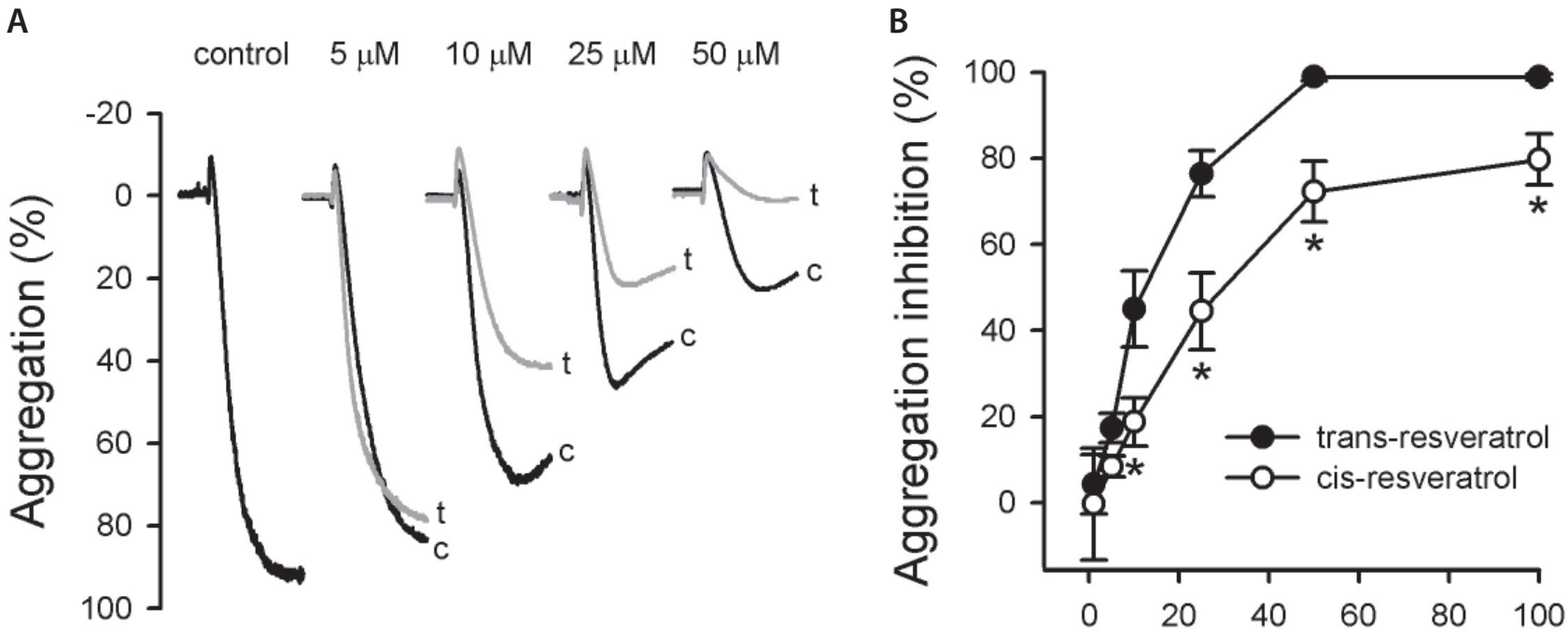

Resveratrol $(\mu \mathrm{M})$

Fig. 2. Inhibition of thrombin-induced platelet aggregation by resveratrol. Platelets were treated with either cis- or trans-resveratrol for 3 $\mathrm{min}$, and aggregation was elicited by $0.2 \mathrm{U} / \mathrm{ml}$ thrombin. Representative tracings of platelet aggregation are presented in $(A)$ and the concentration-dependent inhibitory effect is plotted in (B). Values are means \pm SE of 5-8 independent experiments. *Significantly different from the groups treated with trans-resveratrol $(p<0.05)$. c: cis-resveratrol, t: trans-resveratrol.

anti-cancer activity (Pettit et al., 2002) and elevated cytosolic calcium in vascular myocytes more potently (Campos-Toimil et al., 2005; Campos-Toimil et al., 2007). Recently, Rius et al. (2010) tested the inhibitory effect of cis- and trans-resveratrol against angiotensin II (Angll)-mediated vascular inflammation and found only the trans-isomer to be effective, although it is not clear whether such a result is caused by a difference in potency or is due to different mechanisms (Rius et al., 2010). Taken together, they allow us to hypothesize that the effect on platelet activity may also be different between cis- and transresveratrol. Hence, this study was designed and performed to investigate the effect of cis-resveratrol on platelet aggregation and to compare it with the effect of the trans-isomer.

\section{MATERIALS AND METHODS}

\section{Materials}

Both cis- and trans-resveratrol were purchased from Cayman Chemical Co. (Ann Arbor, MI, USA). Collagen and ADP were from Chrono-log Co. (Havertown, PA, USA) and thrombin was obtained from Sigma-Aldrich Co. (St. Louis, MO, USA). All other chemicals used were of the highest purity available and purchased from standard suppliers.

\section{Animals}

All animal experiments were conducted in accordance with protocols approved by the Ethics Committee of the Animal Service Center at Chonnam National University. Male Sprague-Dawley rats weighing $150-250 \mathrm{~g}$ were purchased from Daehan Biolink (Eumseong, Korea). Prior to experiments, animals were acclimated for 1 week in the laboratory animal facility and maintained at constant temperature and humidity with a 12-hr light/dark cycle. Food and water were provided ad libitum.

\section{Preparation of platelets and measurement of platelet aggregation}

A platelet aggregation study was performed using a 4-channel aggregometer (Chrono-log, Havertown, PA, USA) as de- 


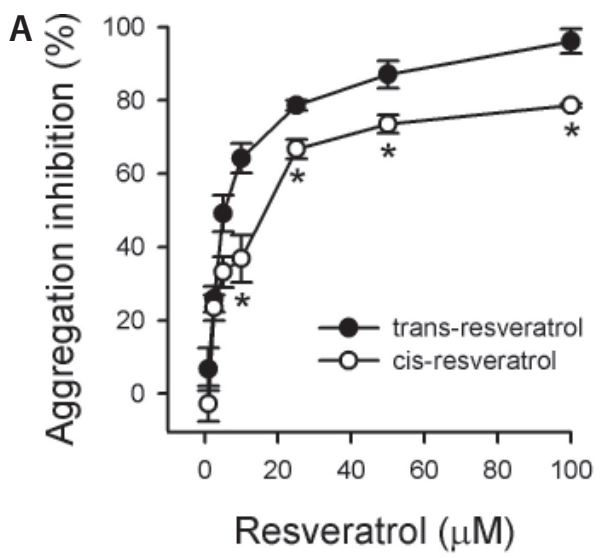

scribed previously (Lee et al., 2002). In brief, blood was collected from the abdominal aorta of ether-anesthetized rats using acid-citrate-dextrose (1:6) as an anticoagulant. After centrifugation at $150 \mathrm{~g}$ for $15 \mathrm{~min}$, platelet-rich plasma (PRP) was obtained from the supernatant, and platelets were isolated by further centrifugation at $1,500 \mathrm{~g}$ for $20 \mathrm{~min}$. Platelets were washed once with washing buffer solution $(138 \mathrm{mM}$ $\mathrm{NaCl}, 2.8 \mathrm{mM} \mathrm{KCl}, 0.8 \mathrm{mM} \mathrm{MgCl}, 0.8 \mathrm{mM} \mathrm{NaH}_{2} \mathrm{PO}_{4}, 10 \mathrm{mM}$ HEPES, 5 mM EDTA, pH 7.4), and were finally resuspended in suspension buffer solution $(138 \mathrm{mM} \mathrm{NaCl}, 2.8 \mathrm{mM} \mathrm{KCl}$, $0.8 \mathrm{mM} \mathrm{MgCl}, 0.8 \mathrm{mM} \mathrm{NaH}_{2} \mathrm{PO}_{4}, 10 \mathrm{mM}$ HEPES, $5.6 \mathrm{mM}$ dextrose, $1 \mathrm{mM} \mathrm{CaCl}, \mathrm{pH} 7.4$ ). The number of platelets was adjusted to $2 \times 10^{8}$ cells $/ \mathrm{ml}$. Platelets were incubated with the indicated concentrations of cis- or trans-resveratrol, and aggregation was induced with sub-maximal concentrations of thrombin, collagen or ADP.

\section{Statistical analysis}

The means and standard errors (SE) of the means were calculated for all experimental groups. The data were subjected to Mann-Whitney test to determine the significance of differences in the effects between cis- and trans-resveratrol. Statistical analysis was performed using SigmaStat software (Version 3.5, Systat Software, San Jose, CA, USA). In all cases, a $p$-value of $<0.05$ was used to determine significance.

\section{RESULTS}

In order to test whether resveratrol isomers inhibited platelet aggregation, platelets were exposed to resveratrol for 3 min, and then aggregation was induced with platelet agonists: thrombin, collagen or ADP. As shown in Fig. 2 and 3, transresveratrol reduced aggregation by thrombin, collagen and ADP in a concentration-dependent manner, as previously reported (Zbikowska et al., 1999; Olas and Wachowicz, 2005). Platelet aggregation could also be inhibited by cis-isomer, and the inhibition was concentration-dependent in the range of 1-100 $\mu \mathrm{M}$ (Fig. 2, 3). However, significant difference was observed in potency between the two isomers, i.e. cis-resveratrol was much less effective than the trans-isomer. $I_{50}$ values of cis- versus trans-isomer were $31 \pm 12$ vs $15 \pm 3,16 \pm 3$ vs 9 \pm 4 , and $60 \pm 15$ vs $25 \pm 6$ for thrombin-, collagen- and ADPinduced aggregation, respectively (Table 1 ). These results indicate that cis-resveratrol is also capable of suppressing the
Table 1. $I C_{50}$ of resveratrol for thrombin-, collagen- or ADP-induced platelet aggregation

\begin{tabular}{lccl}
\hline & \multicolumn{3}{c}{$\mathrm{IC}_{50}(\mu \mathrm{M})^{\mathrm{a}}$} \\
\cline { 2 - 4 } & Thrombin & Collagen & ADP \\
\hline Cis-resveratrol & $31 \pm 12$ & $16 \pm 3$ & $60 \pm 15$ \\
Trans-resveratrol & $15 \pm 3$ & $9 \pm 4$ & $25 \pm 6$ \\
\hline
\end{tabular}

${ }^{a}$ The calculated concentration required for $50 \%$ inhibition of aggregation. Values are expressed as mean \pm SE from 3-8 independent experiments.

response of platelets to proaggregatory stimuli. However, the clinical efficacy as well as the potency of the cis-isomer may not be equal to that of the trans-isomer.

\section{DISCUSSION}

Although extensive attention has been paid to the biological activities of resveratrol, most studies to date have focused on the trans-isomer. Hence, there have been only a limited number of reports comparing cis- and trans-resveratrol content in diets. Recently, a growing number of papers are reporting the quantitative significance of cis-resveratrol in diets (Vitrac et al., 2005; Zamora-Ros et al., 2008). The cis-isomer can be formed from trans-isomer by exposure to ultraviolet radiation (Stivala et al., 2001). cis-Resveratrol is virtually not detected in grapes, but it is present in wines at variable concentrations, which has been ascribed to its production from trans-resveratrol by yeast isomerases during fermentation (Soleas et al., 1997; Fremont, 2000; Cvejic et al., 2010). Given these studies, it is obvious that cis-resveratrol, like the trans-isomer, is also available through the diet and is capable of exerting its clinical effects. It is, therefore, of consequence to characterize the biological activities of cis-resveratrol.

There are only a few comparative studies that reported differences in biological functions between cis- and trans-resveratrol (Orallo, 2006). Such differences have been observed both in vitro and in vivo, including its anti-cancer effect (Pettit et al., 2002), calcium-elevating activity in vascular myocytes, and suppression of Angll-mediated vascular inflammation (Campos-Toimil et al., 2005; Campos-Toimil et al., 2007). There was 
also a difference in metabolic rate through glucuronidation, i.e. glucuronidation occurred at a faster rate with cis-resveratrol (Aumont et al., 2001). Most of the findings appear, to date, to be a quantitative difference rather than a qualitative one between the two isomers. Here, the results of our study provide the novel findings that the anti-platelet effect of cis-isomer is not as potent as that of the trans-isomer.

In this study, cis-resveratrol, like the trans-isomer, suppresses platelet aggregation induced by proaggregatory stimuli such as collagen, ADP and thrombin. However, the potency of the cis-isomer was significantly lower than that of the trans-isomer, the cis-isomer showing an approximately 2-fold higher $I_{50}$. Bertelli et al. (1996) tested the anti-aggregatory effect of cis-resveratrol with collagen. Although cis-resveratrol was effective, as shown here, they did not find any difference between the two isomers, which is not consistent with the results of our study (Bertelli et al., 1996). It might be due to the simplicity of their experiments. In their short paper, they compared only one concentration of resveratrol and did not test a broad concentration range, which might allow them to miss concentrations that do show a difference.

The anti-platelet effect of trans-resveratrol has been well demonstrated through extensive studies and diverse biological activities have been suggested as mechanisms (Olas and Wachowicz, 2005). However, it is complicated and still unclear as the direct target molecules have never been identified despite the fact that so many mechanisms are known to be involved. Accordingly, it may not be simple to elucidate the reason why the cis-isomer is less potent than the trans-isomer. Currently, the underlying mechanism remains elusive and it is not even clear whether both isomers share a common mode of action. Further study will be required to clarify the mechanisms underlying the anti-platelet effect of cis-resveratrol.

\section{ACKNOWLEDGMENTS}

This study was financially supported by Chonnam National University, 2007.

\section{REFERENCES}

Aumont, V., Krisa, S., Battaglia, E., Netter, P., Richard, T., Merillon, J. M., Magdalou, J. and Sabolovic, N. (2001) Regioselective and stereospecific glucuronidation of trans- and cis-resveratrol in human. Arch. Biochem. Biophys. 393, 281-289.

Bertelli, A. A., Giovannini, L., Bernini, W., Migliori, M., Fregoni, M., Bavaresco, L. and Bertelli, A. (1996). Antiplatelet activity of cis-resveratrol. Drugs Exp. Clin. Res. 22, 61-63.

Campos-Toimil, M., Elies, J., Alvarez, E., Verde, I. and Orallo, F. (2007). Effects of trans- and cis-resveratrol on $\mathrm{Ca}^{2+}$ handling in A7r5 vascular myocytes. Eur. J. Pharmacol. 577, 91-99.

Campos-Toimil, M., Elies, J. and Orallo, F. (2005) Trans- and cisresveratrol increase cytoplasmic calcium levels in A7r5 vascular smooth muscle cells. Mol. Nutr. Food Res. 49, 396-404.

Crescente, M., Jessen, G., Momi, S., Holtje, H. D., Gresele, P., Cerletti, C. and de Gaetano, G. (2009) Interactions of gallic acid, resveratrol, quercetin and aspirin at the platelet cyclooxygenase-1 level Functional and modelling studies. Thromb. Haemostasis. 102, 336-346.

Cvejic, J. M., Djekic, S. V., Petrovic, A. V., Atanackovic, M. T., Jovic, S. M., Brceski, I. D. and Gojkovic-Bukarica, L. C. (2010) Determination of trans- and cis-resveratrol in Serbian commercial wines. J. Chromatogr. Sci. 48, 229-234.
Dobrydneva, Y., Williams, R. L. and Blackmore, P. F. (1999) transResveratrol inhibits calcium influx in thrombin-stimulated human platelets. Brit. J. Pharmacol. 128, 149-157.

Fremont, L. (2000) Biological effects of resveratrol. Life Sci. 66, 663673.

Gresele, P., Pignatelli, P., Guglielmini, G., Carnevale, R., Mezzasoma, A. M., Ghiselli, A., Momi, S. and Violi, F. (2008) Resveratrol, at concentrations attainable with moderate wine consumption, stimulates human platelet nitric oxide production. J. Nutr. 138, 1602-1608.

Lee, M. Y., Bae, O. N., Chung, S. M., Kang, K. T., Lee, J. Y. and Chung, J. H. (2002) Enhancement of platelet aggregation and thrombus formation by arsenic in drinking water: a contributing factor to cardiovascular disease. Toxicol. Appl. Pharmacol. 179, 83-88.

Leiro, J., Alvarez, E., Arranz, J. A., Laguna, R., Uriarte, E. and Orallo, F. (2004) Effects of cis-resveratrol on inflammatory murine macrophages: antioxidant activity and down-regulation of inflammatory genes. J. Leukoc. Biol. 75, 1156-1165.

Olas, B. and Wachowicz, B. (2005) Resveratrol, a phenolic antioxidant with effects on blood platelet functions. Platelets 16, 251-260.

Olas, B., Wachowicz, B., Holmsen, H. and Fukami, M. H. (2005) Resveratrol inhibits polyphosphoinositide metabolism in activated platelets. BBA-Biomembranes 1714, 125-133.

Olas, B., Wachowicz, B., Saluk-Juszczak, J., Zielinski, T., Kaca, W. and Buczynski, A. (2001a) Antioxidant activity of resveratrol in endotoxin-stimulated blood platelets. Cell Biol. Toxicol. 17, 117-125.

Olas, B., Wachowicz, B., Szewczuk, J., Saluk-Juszczak, J. and Kaca, W. (2001b) The effect of resveratrol on the platelet secretory process induced by endotoxin and thrombin. Microbios 105, 7-13.

Olas, B., Zbikowska, H. M., Wachowicz, B., Krajewski, T., Buczynski, A. and Magnuszewska, A. (1999) Inhibitory effect of resveratrol on free radical generation in blood platelets. Acta. Biochim. Pol. 46, 961-966.

Orallo, F. (2006) Comparative studies of the antioxidant effects of cisand trans-resveratrol. Curr. Med. Chem. 13, 87-98.

Pettit, G. R., Grealish, M. P., Jung, M. K., Hamel, E., Pettit, R. K., Chapuis, J. C. and Schmidt, J. M. (2002) Antineoplastic agents. 465. Structural modification of resveratrol: sodium resverastatin phosphate. J. Med. Chem. 45, 2534-2542.

Rius, C., Abu-Taha, M., Hermenegildo, C., Piqueras, L., Cerda-Nicolas, J. M., Issekutz, A. C., Estan, L., Cortijo, J., Morcillo, E. J., Orallo, F. and Sanz, M. J. (2010) Trans- but not cis-resveratrol impairs angiotensin-II-mediated vascular inflammation through inhibition of NF-kappaB activation and peroxisome proliferator-activated receptor-gamma upregulation. J. Immunol. 185, 3718-3727.

Shen, M. Y., Hsiao, G., Liu, C. L., Fong, T. H., Lin, K. H., Chou, D. S. and Sheu, J. R. (2007) Inhibitory mechanisms of resveratrol in platelet activation: pivotal roles of p38 MAPK and NO/cyclic GMP. Brit. J. Haematol. 139, 475-485.

Soleas, G. J., Diamandis, E. P. and Goldberg, D. M. (1997) Resveratrol: a molecule whose time has come? And gone? Clin. Biochem. 30, 91-113.

Stivala, L. A., Savio, M., Carafoli, F., Perucca, P., Bianchi, L., Maga, G., Forti, L., Pagnoni, U. M., Albini, A., Prosperi, E. and Vannini, V. (2001) Specific structural determinants are responsible for the antioxidant activity and the cell cycle effects of resveratrol. J. Biol. Chem. 276, 22586-22594.

Vitrac, X., Bornet, A., Vanderlinde, R., Valls, J., Richard, T., Delaunay, J. C., Merillon, J. M. and Teissedre, P. L. (2005) Determination of stilbenes (delta-viniferin, trans-astringin, trans-piceid, cis- and trans-resveratrol, epsilon-viniferin) in Brazilian wines. J. Agric. Food Chem. 53, 5664-5669.

Yang, Y. M., Chen, J. Z., Wang, X. X., Wang, S. J., Hu, H. and Wang, H. Q. (2008a) Resveratrol attenuates thromboxane A2 receptor agonist-induced platelet activation by reducing phospholipase C activity. Eur. J. Pharmacol. 583, 148-155.

Yang, Y. M., Wang, X. X., Chen, J. Z., Wang, S. J., Hu, H. and Wang, H. Q. (2008b) Resveratrol attenuates adenosine diphosphate-induced platelet activation by reducing protein kinase $\mathrm{C}$ activity. Am. J. Chinese Med. 36, 603-613.

Zamora-Ros, R., Andres-Lacueva, C., Lamuela-Raventos, R. M., Berenguer, T., Jakszyn, P., Martinez, C., Sanchez, M. J., Navarro, C., Chirlaque, M. D., Tormo, M. J., Quiros, J. R., Amiano, P., Dor- 
Kim et al. Anti-Platelet Effect of cis- and trans-Resveratrol

ronsoro, M., Larranaga, N., Barricarte, A., Ardanaz, E. and Gonzalez, C. A. (2008) Concentrations of resveratrol and derivatives in foods and estimation of dietary intake in a Spanish population: European Prospective Investigation into Cancer and Nutrition (EPIC)Spain cohort. Br. J. Nutr. 100, 188-196.
Zbikowska, H. M. and Olas, B. (2000) Antioxidants with carcinostatic activity (resveratrol, vitamin $\mathrm{E}$ and selenium) in modulation of blood platelet adhesion. J. Physiol. Pharmacol. 51, 513-520.

Zbikowska, H. M., Olas, B., Wachowicz, B. and Krajewski, T. (1999) Response of blood platelets to resveratrol. Platelets 10, 247-252. 